\title{
Design of Large-scale Platform Detection System Based on Distributed Wireless Communication Technology
}

\author{
Peng $\mathrm{Li}^{1,2}$, Dengpei $\mathrm{Ji}^{1,2}$, Hao Liu ${ }^{1,2}$, Wenli $\mathrm{Xu}^{1,2}$ \\ 1.Tianjin Institute of Aerospace Mechanical and Electrical Equipment, Tianjin 300301, China \\ 2. Tianjin Key lab of Microgravity and Hypogravity Environment Simulation Technology, Tianjin 300301, China \\ jdp503@163.com, lipenglp0805@163.com, liuhao1992111@126.com, xuwenli_1988@126.com
}

\begin{abstract}
Aiming at the problem that the traditional detection device can not avoid obstacles and easily collides with the equipment on the platform during the detection of the large-scale ultra-flat support platform, a detection scheme of large-scale ultra-flat support platform based on distributed wireless communication technology is proposed in this paper. Using special measuring instruments combined with embedded system hardware to quickly build a distributed detection system, measuring data through the wireless network communication to the control computer under the stage to achieve efficient automatic measurement, while measuring the platform support mechanism adjustment. The platform level and height difference measurement data act as a support mechanism of the feedback to achieve measurement and regulation of closed-loop control.
\end{abstract}

Keywords-Wireless communication; large-scale platform; detection

\section{INTRODUCTION}

Large-scale ultra-flat support platform is the large spacecraft experimental platform which composed of dozens of granite block plate combination. In order to ensure that the level of the entire plane to meet the requirements, the granite slab should adjust the levelness between each other, so the measurement of relative levelness is particularly important. In the experiment, usually using the automatic tracing detection vehicle to complete the relative levelness measurement, but the platform is usually equipped with immobile experimental equipment, automatic tracing test car which in the testing process is very easy to bump into the experimental equipment, so that it also affect the normal detection work. Increasing obstacle avoidance modules for the vehicle can add significant cost. The use of special measuring instruments combined with embedded system hardware to quickly build a distributed wireless transmission detection system, not only can reduce the existing costs and reduce unnecessary workload, but also to ensure the adjustment work of levelness quickly and safely.

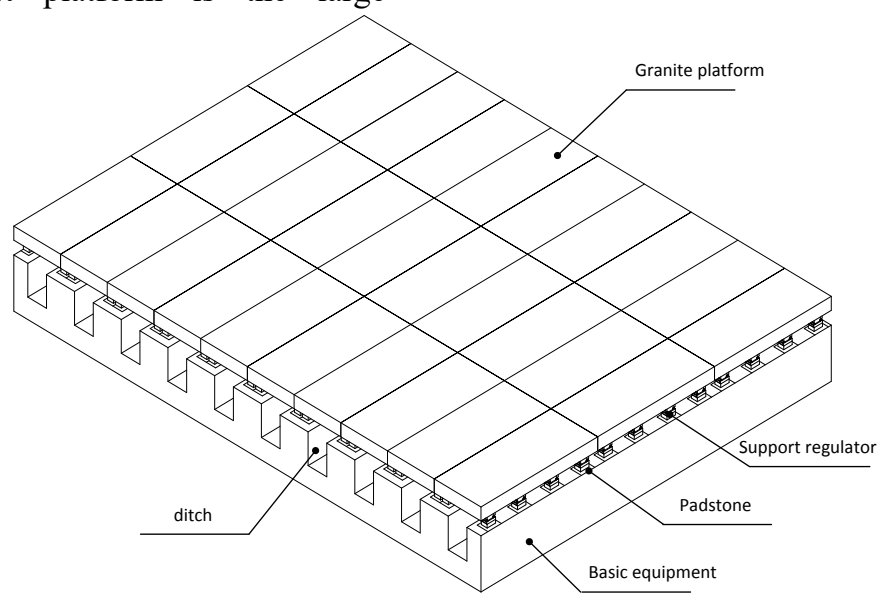

Fig. 1. Schematic diagram of platform structure

Wireless transmission and wireless networking technology using WIFI methods. WIFI is a radio signal that is used for short-range transmission and can access the Internet using the IEEE802.11 protocol over a few hundred feet. The physical layer and control layer of media access are defined in the first version released in 1997, and stipulate the basic transmission medium and basic network structure for wireless LAN, and In 1999 adding the IEEE802.1la and IEEE802.1lg standards. Because of its transfer rate can reach $54 \mathrm{Mbp} / \mathrm{s}$, it can support the real-time transmission of measurement data requirements. 


\section{THE OVERALL DESIGN OF THE SYSTEM}

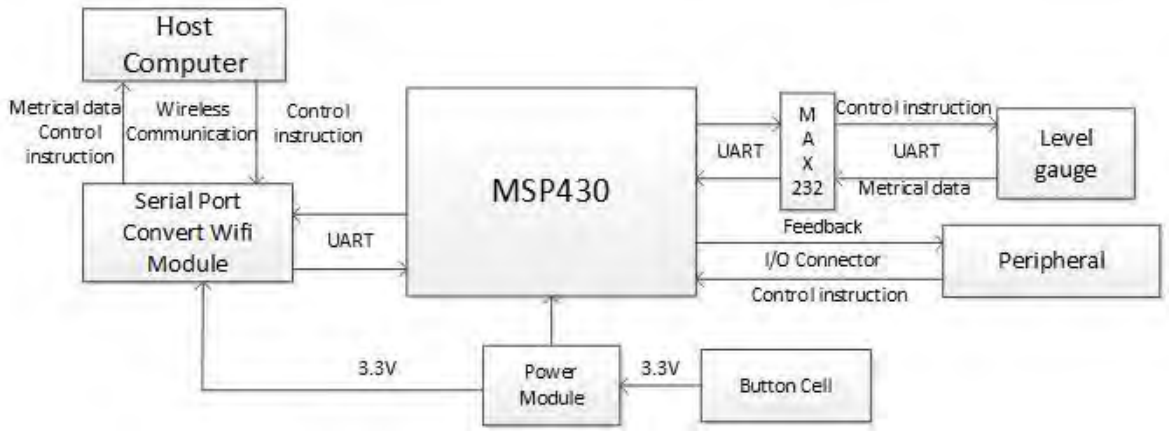

Fig. 2. Detection system structure

From the overall system design plan view, the system modules exchange information through the serial port, and complete data exchange between the data source and the host computer through the wireless communication transmission. The system adopts the MSP430 series microcomputer, which has outstanding performance in low-power area as the control core. MSP430 is responsible for processing the collected levelness data and driving the serial port communication function. The entire system through a number of peripheral control devices to complete the measurement process, including the data collection、 access to the current state and other control functions.

Data transmission, the Level Check as a data source, and the serial-to-WIFI module as a data upload. The data transfer can be realizing by using serial communication between them and master chip microcontroller; at the same time, the microcontroller can also communicate with them through the serial port and send command to them, so that they can achieve other functions in the system better. Through the WIFI wireless communication, complete the data uploading、 mode configuration and other interactive actions will be completed between serial-to-WIFI module and the host computer.

The system is powered by one or more replaceable batteries and is converted to $3.3 \mathrm{~V}$ standard voltage by power module. It will process to power the microcontroller, serial-to-WIFI module, and other supporting chips.

\section{THE DESIGN OF THE SYSTEM HARDWARE}

\section{A. The Design of The Minimum System for The Main}

\section{Control Chip of The Measurement and Control System}

Data reception and transmission are done through the serial port communication, so the master chip needs to have two serial ports at least. The start and stop of the measurement process and the control of the function selection are all done by the peripheral equipment, so the microcontroller should need to have more abundant $\mathrm{I} / \mathrm{O}$ interfaces which can be connected with the external equipment. At the same time, the system is powered by batteries rather than a continuous power supply, so there is the need of low energy consumption for the entire system. Based on the above requirements, MSP430F149 microcontroller, which is produced by Texa Instruments, is selected as the main control chip of the whole measurement and control system.

MSP430F149 typical pin definition and the hardware connection diagram of the minimum system has been shown in Figure 2-1:

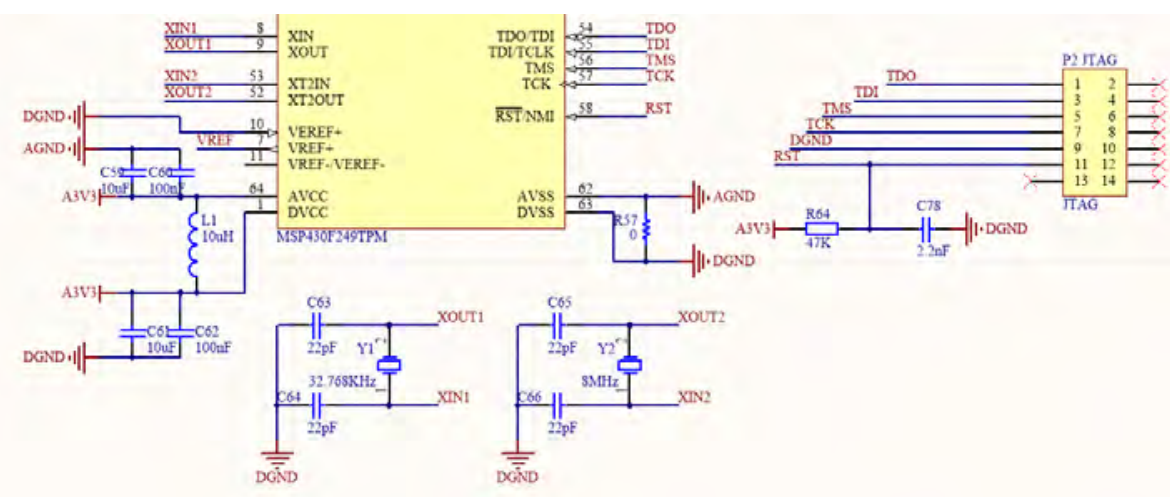

Fig. 3. The minimum system for MSP430F149

Among them, a $10 \mu \mathrm{H}$ inductance has been connected between the analog power and digital power. Also, a $0 \mathrm{Ohm}$ resistor which is used to distinguish between analog and digital power supply has been accessed between the analog 
ground and digital ground. And at the same time, the system interference will be reduced. The system uses two kinds of crystal oscillator to produce system clocks. One kind is the crystal which frequency is $32.768 \mathrm{KHz}$, and the other kind is the crystal which frequency is $8 \mathrm{MHz}$. When using the $8 \mathrm{MHz}$ system clock, system's speed can be greatly enhanced because of the high command speed which is up to 8MIPS.On the other hand, although the system running slower when using $32.768 \mathrm{KHz}$ system clock, the clock can be used for serial port configuration and other occasions, so that the application of the microcontroller become more flexible. A JTAG interface bas been set as a system programming interface. It will not only adjust the system under the real-time conditions at any time, but also greatly simplifies the debugging process.

\section{B. Design of Data Communication Circuit of Measurement}

\section{And Control System}

The data transmission between the Level Check and the system, which also happens between the wireless module and the microcontroller, is accomplished through the serial port communication. The serial port 0 of the microcontroller is used to send instructions to the Level Check and receive the measurement data sent by it. The serial port 1 of the microcontroller is used to send the measurement data to the wireless module which will be uploaded. Therefore, the design of the communication circuit can be divided into two parts: the downward part connected with the Level Check and the upward part connected with the wireless module. Figure 2-2 shows the hardware connection of the downward part:
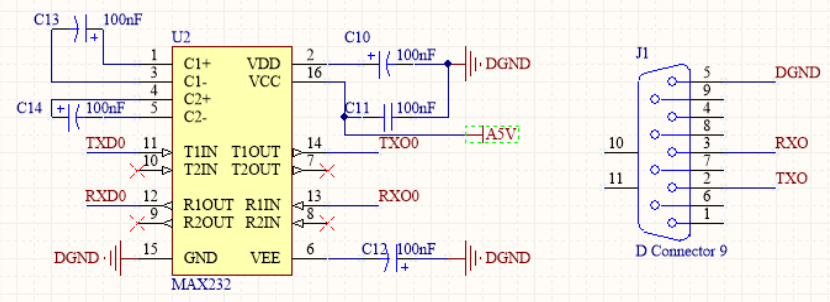

Fig. 4. The circuit of serial communication chip

Wireless module integrates MAC, baseband chip, RF transceiver unit, and power amplifier; built-in low-power operation mechanism that can effectively achieve low-power module operation; support WIFI protocol and TCP/IP protocol. The user only need simple configuration, the UART networking equipment can be achieved. Smaller in size, it is easy to assemble on the hardware veneer circuit of customer's products. And the size is small, so that it is easy to assemble on the hardware circuit of customer's products. Figure 2-3 shows the connection diagram:

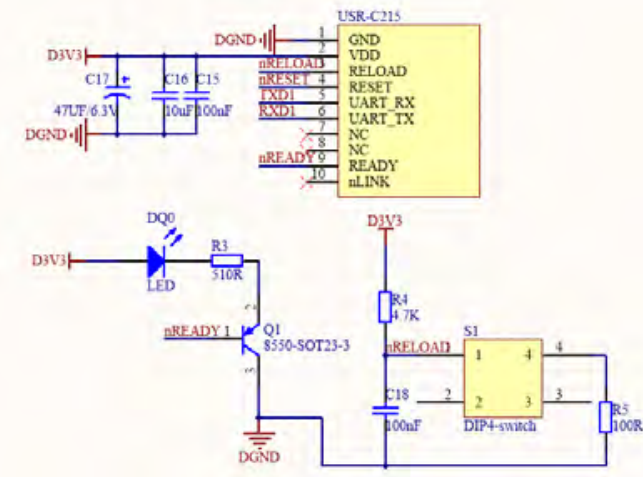

Fig. 5. The circuit of Wireless communication module

The serial port 1 of the microcontroller connect with the serial port pin of the wireless module crosswise, the wireless communication module adopts the transparent transmission mode whose figure is plug and play, thus minimize the complexity of user usage. When in the transparent transmission mode, the user only need to configure the necessary parameters, and then can communicate with the network through the UART interface. After power-on, the module automatically connects to the configured wireless network and server. This mode mainly implements the user equipment data and fixed server to interact, through a simple socket communication. The working mode as the figure 6 shown:

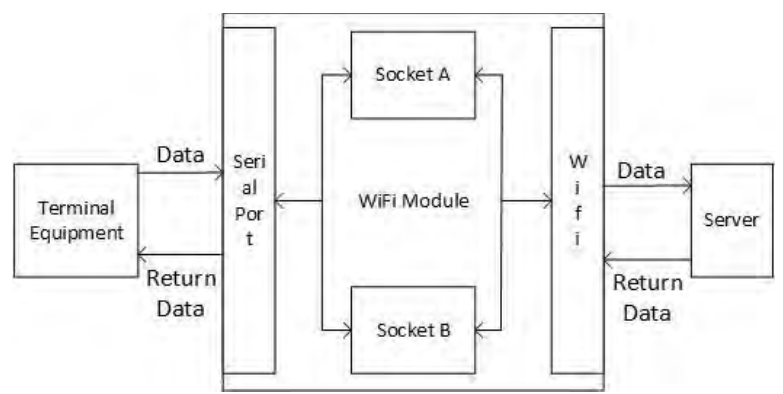

Fig. 6. The working mode of wireless communication module

The module has a serial port 0 can carry on the data transparent transmission with Socket A and Socket B. When the module works in the transparent mode, the data written to the UART interface of the module will be automatically sent to the two sockets; the data received by the module through the Socket are all sent through the UART and WIFI interface.

In addition, the wireless communication module uses the STA mode to carry out the wireless networking, and the schematic diagram of the network connection structure is shown in Figure 7 below.

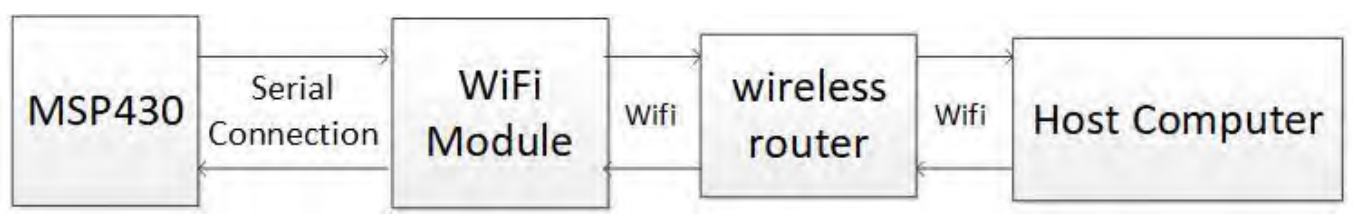

Fig. 7. Wireless networking 
STA is a wireless access point, and it's also a wireless network terminal. The network mode of the distributed measurement system is STA mode, it means that the wireless router is in the center and the mutual communication among all the STAs is completed by the wireless router. Since there are many wireless modules in the distributed measurement system, you need to set the IP address of each wireless module configuration and then set the server address and port number to access the same central router. At the same time, setting the monitoring host server address, and then it can achieve the wireless module to send the data acquisition.

\section{Source Module}

The system uses button batteries for power supply. However, the voltage of batteries does not meet the voltage range requirements of each module in the system. The voltage needs to be converted to meet the power supply requirements of the system. In this design, in order to meet the system's voltage and current needs, MIC29302 voltage converter, which is a kind of low-voltage linear regulator power supply and its maximum output current up to $3 \mathrm{~A}$,produced by the MICREL company has been chosen. The output voltage of MIC29302 can be adjusted, while a variety of fixed output version.3.3V fixed output version is used in this design. Figure 2-6 shows the hardware connection of the power module:

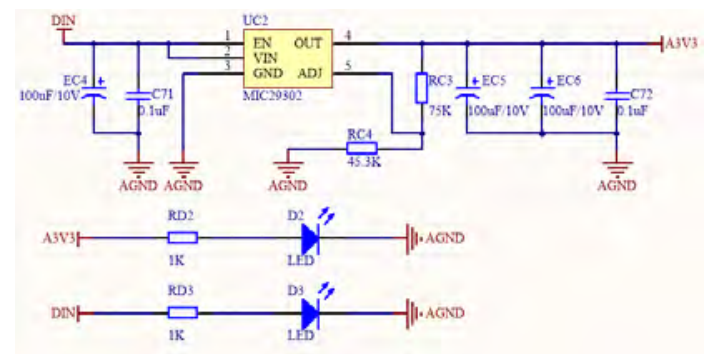

Fig. 8. The circuit of Power module

System uses two to several batteries for power supply, the power supply voltage DIN as MIC29302's input voltage. The MIC29302 requires only a capacitor configuration and a simple resistor-ratio configuration to output a stable $3.3 \mathrm{~V}$ output voltage as the supply voltage for each module in the system.

Although the MSP430 has two kinds of power which are analog power and digital power, but the design does not need to use MSP430 analog signal processing part, so there is no need to use two power chips to separate analog power and digital power completely. So we only use the method described in section 2.1 when establish the microcontroller minimum system.

\section{THE DESIGN OF THE SYSTEM SOFTWARE}

The software part of the system is written by $\mathrm{C}$ language. It has the characteristics of good portability and strong structure, which makes the debugging, testing and maintenance of the program easy. The way of programming of microcontrollers is executed on the polling and interrupt.
The characteristic of polling is that the controller executes each instruction in the main loop one by one. However, not all instructions are used in actual application, which not only slows down the execution of the entire program but also causes additional energy consumption. The interrupt can reduce the internal resource consumption of the controller to improve real-time system response, and in the implementation of the corresponding operation to release the thread and then return to low-power mode, So that it can play MSP430 low power consumption advantages and improve the overall system life. The flow chart of the program is shown in the following diagram.

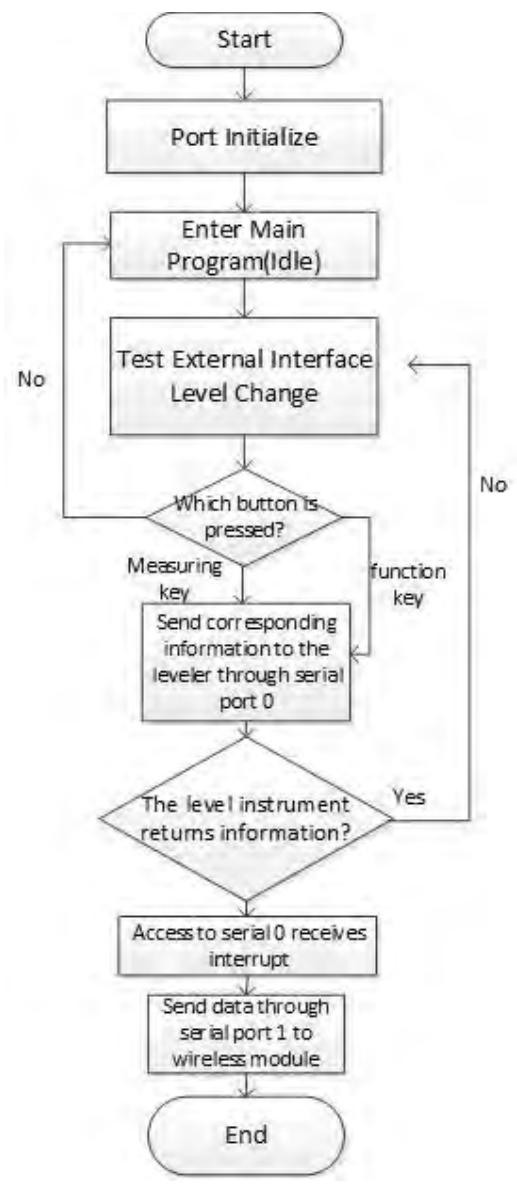

Fig. 9. The flow chart of the program

After the system is powered on, the MSP430 automatically executes the programmed program from the internal Flash of the chip. In the initial stage, the MSP430 will turn off the watchdog, initialize the clock, initialize the I/O ports and the corresponding serial port configuration settings. After all complete, the program will enter the main part. This initialization part will only run once every time the system is powered on, and will not be repeated.

After entering the main program, the program will first detect whether the peripheral devices connected to the system are pressed or not, which will be reflected in the level of the microcontroller I / O interface connected to the peripheral devices. If a change is detected, the MSP430 will determine which peripheral device is triggered based on the $\mathrm{I} / \mathrm{O}$ port 
number and call Serial 0 send programe to send the appropriate command to the Level Check. Normally, the Level Check receives commands through the serial port, it will immediately return data information to the MSP430 through the serial port.

In order to save resources and reduce energy consumption, the serial port receive operations using interrupt handling. If the Level Check did not return data to the system, serial port 0 receive interrupt will not be triggered, the program will roll back to the idle state. When Level Check return data to the system, the receive interrupt operations of serial port will be triggered, interrupt program will receive data in the serial port 0 receive cache, and then sent to the serial port 1 to be sent. When the send operation of serial port 1 is completed, the main program is completely executed once, and roll back to the beginning of the main program. Since the main program is in the while loop, so it can wait for the next cycle of execution.

\section{TEST RESULTS AND ANALYSIS}

In order to verify the reliability of the system, the whole system was tested. In order to observe the uploading information of the system more intuitively, using LABVIEW to prepare the host computer display interface. During the measurement process, the digital display Level Check is randomly placed at 4 measurement points, and the level difference displayed on the level indicator and the level difference displayed by the host computer are compared every 6 consecutive seconds. Table 1 shows the comparison of measurement data:

TABLE I. The Results of The Test

\begin{tabular}{|c|c|c|c|c|c|c|c|c|}
\hline \multirow{2}{*}{ Time } & \multicolumn{2}{|c|}{ TEST 1 } & \multicolumn{2}{c|}{ TEST 2 } & \multicolumn{2}{c|}{ TEST 3 } & \multicolumn{2}{c|}{ TEST 4 } \\
\cline { 2 - 9 } & Terminal & Host & Terminal & Host & Terminal & Host & Terminal & Host \\
\hline $1 \mathrm{~s}$ & $4.16^{\prime \prime}$ & $4.16^{\prime \prime}$ & $4.11^{\prime \prime}$ & $4.11^{\prime \prime}$ & $4.15^{\prime \prime}$ & $4.15^{\prime \prime}$ & $4.13^{\prime \prime}$ & $4.13^{\prime \prime}$ \\
\hline $2 \mathrm{~s}$ & $4.15^{\prime \prime}$ & $4.15^{\prime \prime}$ & $4.10^{\prime \prime}$ & $4.10^{\prime \prime}$ & $4.15^{\prime \prime}$ & $4.15^{\prime \prime}$ & $4.13^{\prime \prime}$ & $4.13^{\prime \prime}$ \\
\hline $3 \mathrm{~s}$ & $4.15^{\prime \prime}$ & $4.15^{\prime \prime}$ & $4.10^{\prime \prime}$ & $4.10^{\prime \prime}$ & $4.15^{\prime \prime}$ & $4.15^{\prime \prime}$ & $4.13^{\prime \prime}$ & $4.13^{\prime \prime}$ \\
\hline $4 \mathrm{~s}$ & $4.15^{\prime \prime}$ & $4.15^{\prime \prime}$ & $4.11^{\prime \prime}$ & $4.11^{\prime \prime}$ & $4.15^{\prime \prime}$ & $4.15^{\prime \prime}$ & $4.12^{\prime \prime}$ & $4.12^{\prime \prime}$ \\
\hline $5 \mathrm{~s}$ & $4.16^{\prime \prime}$ & $4.16^{\prime \prime}$ & $4.11^{\prime \prime}$ & $4.11^{\prime \prime}$ & $4.15^{\prime \prime}$ & $4.15^{\prime \prime}$ & $4.12^{\prime \prime}$ & $4.12^{\prime \prime}$ \\
\hline $6 \mathrm{~s}$ & $4.16^{\prime \prime}$ & $4.16^{\prime \prime}$ & $4.11^{\prime \prime}$ & $4.11^{\prime \prime}$ & $4.15^{\prime \prime}$ & $4.15^{\prime \prime}$ & $4.13^{\prime \prime}$ & $4.13^{\prime \prime}$ \\
\hline
\end{tabular}

It can be seen from the table that the measurement result of the level difference which is uploaded to the host computer via the system basically agrees with the display value of the Level Check itself, so that the design principle of the system is correct and the measurement accuracy is high.

\section{CONCLUSION}

This paper presents a detection scheme based on distributed wireless communication technology for large ultra-flat support platform. The system mainly consists of two parts: measurement and wireless transmission. The use of special measuring instruments placed in the designated location, the measurement part to obtain and process the level data, and then the data will be transmitted to host computer through wireless. So that it can achieve efficient automatic measurement. The test prove that the closed-loop control of measurement and regulation is stable and reliable, which proves that the whole system has the correct principle and complete functions. The system solves the problem of inconvenience and high cost of large-scale platform in complex environment, and at the same time, the design goal of getting the level data of large platform correctly and conveniently is achieved.

\section{ACKNOWLEDGMENTS}

This work was supported by the National Natural Science Foundation of China under Grant No.51705363 and the key technologies R \& D program of Tianjin under Grant No.14RCHZGX00874.

\section{REFERENCES}

[1] Xiaoming Mo, Jing Chen, Smart Home Wireless Networking Technology. Microprocessors, 2007(01).

[2] Shaoying Xiong, The Design and Implementation of WIFI Share System. Xi'An, Xidian University, 2012.

[3] Changda Wang, Shiguang Ju, Security in Wireless Networking Technology. Computer Science, 2006(07).

[4] Xiaoyang Li, The Application of WIFI Wireless Interconnection Technology in Smart Home. Paper of Electric, 2012,9.

[5] Hui Chen, Cinan Wu, Design of Wireless Networking Module for Greenhouse Environment Monitoring Based on Si4432. Microcontrollers \& Embedded Systems, 2018(1). 